\title{
Casimir energy and entropy between dissipative mirrors
}

\author{
F. Intravaia and C. Henkel \\ Universität Potsdam, Institut für Physik, Am Neuen Palais 10, 14469 Potsdam, Germany \\ E-mail: francesco.intravaia@qipc.org
}

\begin{abstract}
We discuss the Casimir effect between two identical, parallel slabs, emphasizing the role of dissipation and temperature. Starting from quite general assumptions, we analyze the behavior of the Casimir entropy in the limit $T \rightarrow 0$ and link it to the behavior of the slab's reflection coefficients at low frequencies. We also derive a formula in terms of a sum over modes, valid for dissipative slabs that can be interpreted in terms of a damped quantum oscillator.
\end{abstract}

\section{Introduction}

In recent years, the Casimir force [1] has gained the status of a mesoscopic force, attracting considerable attention from physicists and engineers who work with nano- or microelectromechanical devices [2, 3, 4]. In particular in view of these applications, many theoretical efforts are addressing more realistic configurations. But also for fundamental applications, a precise knowledge of experimentally relevant imperfections is valuable to tighten upper limits [5, 6]. The Casimir effect originally describes the force between two parallel, perfectly reflecting slabs placed in vacuum at zero temperature, and it is quite clear that the generalization to a realistic experimental setup roughly articulates in three steps: (i) realistic materials, (ii) non-zero temperature, and (iii) other geometries. Step (i) was essentially achieved by Lifshitz in 1955 [7], and within the same theory, addressing step (ii) was apparently trivial. Surprisingly, up to now, the role of dissipation in the temperature correction to the force between two dissipative metallic slabs is unclear and at the center of hottest controversy around the Casimir effect [8, 9, 10, 11, 12, 13, 14, 15]. Lifshitz adopts a "matter" point of view to calculate the Casimir effect building on a stochastic (Langevin) version of the Maxwell equations where quantum fluctuating currents are responsible for the fields. Although the inclusion of dissipation is quite natural in this theory, it becomes rather complicated when we adopt a "field" point of view. The problem is that the eigenfrequencies of the field, $\omega_{m}$, become complex with absorbing mirrors, so that the expression used by Casimir

$$
E=\frac{\hbar}{2}\left[\sum_{m} \omega_{m}\right]_{\infty}^{L}
$$

is meaningless. We use the symbol $[\cdots]_{\infty}^{L}$ to denote the renormalization of the sum over mode branches $m$, subtracting the limiting value of the square bracket when the mirror distance $L \rightarrow \infty$. Casimir's expression (1) is directly generalizable to partially reflecting (dispersive) mirrors [16], but it reveals to be rather tricky in the dissipative case. Among 
others, Langbein [17, 18] has suggested that one should take the real part of the complex energy, but his heuristic arguments remain unsatisfactory.

In this paper we discuss two different aspects of including dissipation into the Casimir effect. We start with a rather general viewpoint on the Casimir entropy at finite temperature, recover previous results and give a criterion to classify them. We also show how Eq.(1) must be modified when complex frequencies enter the theory, in order to take consistently into account the dissipation phenomena.

\section{The Casimir entropy}

The current controversy arises when calculating the correction to the Casimir force at finite (nonzero) temperature. Although the calculation was implemented in Lifshitz's pioneering paper and the problem was already recognized by Schwinger et al. [19], it has been carefully examined only recently. An orthodox application of Lifshitz's formula for metal has lead to some doubts concerning the way one describes their optical properties. Indeed, using the Drude model to describe the optical response of a metallic mirror leads to a completely different behavior of the TE and TM polorizations, in stark contrast to perfect reflectors (Dirichlet boundary conditions) that, by definition, perfectly reflect both polarizations. This phenomenon is fairly striking at high temperatures, but it leads to significant thermal corrections also, in particular for the Casimir entropy, in the low temperature limit. In fact, one important thermodynamical principle (the Nernst heat theorem) states that when we deal with an equilibrium situation the system entropy must go to zero in the limit $T \rightarrow 0$.

The final result of Lifshitz's paper is an expression for the Casimir free energy as a sum over Matsubara frequencies:

$$
\mathcal{F}=\frac{\hbar}{2 \pi} \sum_{p, \mathbf{k}} \sum_{n=0}^{\infty}{ }^{\prime} \tau g_{k}^{p}(\mathrm{i} n \tau, \tau)
$$

where $p$ labels the polarization (TM, TE) of an electromagnetic wave with wavevector $\mathbf{k}$ parallel to the slab surface. The primed sum has a prefactor $1 / 2$ in the first term, as usual. The function $g_{k}^{p}(\omega, \tau)=\ln \left[1-r_{\mathbf{k}}^{p}(\omega, \tau)^{2} e^{-2 \kappa L}\right]$ and we use $[\tau$ is the first Matsubara frequency]

$$
\kappa^{2}=k^{2}-\omega^{2} / c^{2}, \quad \tau=2 \pi k_{B} T / \hbar
$$

Note that we have allowed for a dependence on temperature of the reflection coefficients $r_{\mathbf{k}}^{p}(\omega, \tau)$. The Casimir entropy can be derived from the previous expression by differentiation. Because the series converges uniformly in the parameter $\tau$ we can interchange the sum and the derivative. In the following, we drop the sum over the quantum numbers $p$ and $\mathbf{k}$ and set $k_{B}=1$ :

$$
S=-\frac{2 \pi}{\hbar} \frac{d \mathcal{F}}{d \tau}=-\sum_{n=0}^{\infty}\left[g(\mathrm{i} n \tau, \tau)+\mathrm{i} n \tau g_{\omega}(\mathrm{i} n \tau, \tau)+\tau g_{\tau}(\mathrm{i} n \tau, \tau)\right]
$$

where we have defined

$$
g_{\omega}(\omega, \tau) \equiv \partial_{\omega} g(\omega, \tau) \quad \text { and } \quad g_{\tau}(\omega, \tau) \equiv \partial_{\tau} g(\omega, \tau)
$$

Of course the first two terms in (4) are more significant than the third, which is identically zero when the reflection coefficient does not depend on temperature. We ignore the third term for the moment and suppress the second argument for simplicity. The sum over $n$ is re-arranged as

$$
\sum_{n=0}^{\infty} \mathrm{i} n \tau\left[g_{\omega}(\mathrm{i} n \tau)-\frac{g(\mathrm{i} n \tau)-g(\mathrm{i}(n-1) \tau)}{\mathrm{i} \tau}\right]-\frac{1}{2} g(0)
$$


Inside the brackets, we use the Taylor expansion of $g$ to get

$$
g(\mathrm{i}(n-1) \tau)-g(\mathrm{i} n \tau)+\mathrm{i} \tau g_{\omega}(\mathrm{i} n \tau)=\sum_{k \geq 2} g_{\omega^{k}}(\mathrm{i} n \tau) \frac{(-\mathrm{i} \tau)^{k}}{k !}
$$

Therefore the entropy per mode can be rewritten as

$$
S=-\left(\sum_{n \geq 0, k \geq 2} n g_{\omega^{k}}(\mathrm{i} n \tau) \frac{(-\mathrm{i} \tau)^{k}}{k !}-\frac{1}{2} g(0)\right)
$$

Using the analytical properties of the function $g$, we can express the $k$ 'th derivative in $\omega$ by a contour integral along a path $C$ in the upper half complex plane (the path must circle around each $z=\mathrm{i} n \tau$ ) and get

$$
S=-\left(\oint_{C} \sum_{n \geq 0, k \geq 2} \frac{g(z) n(-\mathrm{i} \tau)^{k}}{(z-\mathrm{i} n \tau)^{k+1}} \frac{d z}{2 \pi \mathrm{i}}-\frac{1}{2} g(0)\right)
$$

Exploiting the Euler-MacLaurin formula [20] we have [ $B_{m}$ are the Bernoulli numbers]

$$
\sum_{n \geq 0} \frac{n(-\mathrm{i} \tau)^{k}}{(z-\mathrm{i} n \tau)^{k+1}}=\frac{(-\mathrm{i} \tau)^{k-2}}{z^{k-1}}\left[\frac{1}{k(k-1)}+\sum_{m \geq 1} B_{2 m} \frac{(k+2 m-1) !}{(2 m-1) ! k !}\left(\frac{\tau}{z}\right)^{2 m}\right]
$$

When this is substituted into Eq. (9), we get an exact series expansion of the Casimir entropy in terms of the temperature and of the derivatives of $g(z)$ at $z=0$.

Let us consider now the limit $\tau \rightarrow 0$, still ignoring the explicit temperature dependence of the reflection coefficients. The sum over $k$ in Eq. (9) is approximated by its first term. We then find that the entropy goes to zero:

$$
\tau \rightarrow 0: \quad S \rightarrow-\left(\frac{1}{2} \oint_{C} \frac{g(z)}{z} \frac{d z}{2 \pi \mathrm{i}}-\frac{1}{2} g(0)\right)=0
$$

This result is the same as the one obtained by Böstrom and Sernelius [8, 21] when the dissipation rate in the Drude model does not depend on temperature. The present demonstration has the advantage to apply to a broad class of reflection coefficients that do not depend on temperature, of course. The first terms of the expansion in $\tau$ are given by

$S \approx-\sum_{p, k} \oint_{C} g(z)\left[-\frac{1}{6} \frac{\mathrm{i} \tau}{z^{2}}+\frac{5}{6} \frac{\tau^{2}}{z^{3}}\right] \frac{d z}{2 \pi \mathrm{i}}=\sum_{p, k}\left[\frac{g_{\xi}(0)}{6} \tau+\frac{5}{12} g_{\xi^{2}}(0) \tau^{2}\right]$

where we express the derivatives in terms of the imaginary frequency $\xi=-i \omega$. In the case of the Drude model, fig 1 shows that for the TM polarization, $g(\mathrm{i} \xi)$ monotonously increases with $\xi$. Hence, $g_{\xi}(0)$ is close to zero or positive. The TE polarization behaves differently: it starts from a value $g(0)$ close to zero and shows first a negative slope before increasing and going to zero as $\xi \rightarrow \infty$; this implies a negative contribution to the entropy. This behavior is due to the vanishing of the reflection coefficient $r_{\mathbf{k}}^{\mathrm{TE}}(\omega \rightarrow 0)$ for any finite $\mathbf{k}$. In the general case, the sign of the low-temperature entropy depends of course on the balance between the derivatives of $g$ for both polarizations; it is therefore model-dependent.

Nevertheless, we can make some general considerations, which are valid for a large class of metals at least as long as the local description for the reflection coefficients is applicable. At high frequencies, as long as they are below the plasma frequency, both TM- and TEreflection coefficients are close to unity because the dielectric function is large, giving a good approximation to the perfect conductor limit $\varepsilon=\infty$. This behaviour continues down to zero frequencies in TM-polarization because here, electric fields are dominant and are 


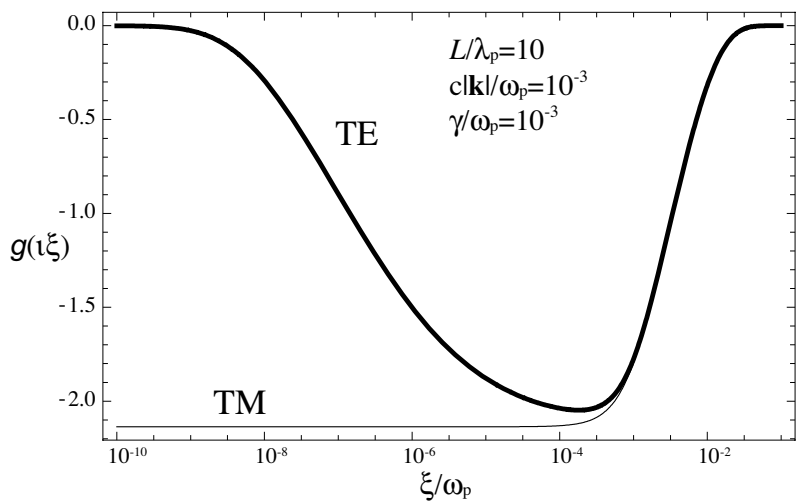

Figure 1. Behavior of $g(\mathrm{i} \xi)$ as function of imaginary frequency $\omega=\mathrm{i} \xi$ for the polarizations TE and TM. The Drude dielectric function is used. See the text for more detail.

efficiently screened by the (Drude) metal. Only for frequencies much larger than plasma frequency $\omega_{\mathrm{p}}$, the reflection coefficient goes quickly to zero. Hence, we expect a small and positive entropy contribution from the TM mode at low temperatures. In TE-polarization, the ultraviolet behavior is the same, but at low frequencies the field is prevalently magnetic and penetrates into the material causing that the reflection coefficient rapidly decreases to zero [22]. This strongly suggests that the TE contribution to the Casimir entropy is negative and larger in magnitude than its TM counterpart. Our analysis also explains why we have always a positive entropy for the plasma (non dissipative) model: the UV limit is unchanged, the behavior of both reflection coefficients is qualitatively the same, and both do not vanish at $\omega \rightarrow 0$. A metal described through the plasma model thus behaves qualitatively similar to a superconductor at sufficiently small frequencies. We can conclude that a negative value of the Casimir entropy is a general feature of the Lifshitz formula when the TE reflection coefficient goes quickly and monotonously to zero in the limit of zero frequency.

Let us consider now a situation where the reflection coefficients $r(\omega, \tau)$ depend on temperature. In this case it could happen that the limits $\tau \rightarrow 0$ and $\omega \rightarrow 0$ do not commute. More specifically,

$$
\lim _{\tau \rightarrow 0} r(\alpha \tau, \tau) \neq \lim _{\tau \rightarrow 0} r(0, \tau) \Rightarrow \lim _{\tau \rightarrow 0} g(\alpha \tau, \tau) \neq \lim _{\tau \rightarrow 0} g(0, \tau)
$$

where $\alpha$ is some non-zero complex constant. This means that $g(\omega, \tau)$ is no longer an analytic function in the whole upper half complex plane when $\omega$ and $\tau$ are not independent and we approach zero along the path $\omega=\alpha \tau$. To illustrate this with more detail, let us consider the Drude model and the Fresnel form of the reflection coefficients:

$$
\varepsilon(\omega, \tau)=1-\frac{\omega_{\mathrm{p}}^{2}}{\omega^{2}[1+\mathrm{i} \gamma(\tau) / \omega]}
$$

where $\gamma(\tau)$ is the (temperature-dependent) dissipation rate. The quantity that plays a crucial role is $\omega^{2} \epsilon(\omega, \tau)$ for which it is easy to see that

$$
\lim _{\tau \rightarrow 0}(\alpha \tau)^{2} \epsilon(\alpha \tau, \tau) \neq \lim _{\tau \rightarrow 0} \lim _{\omega \rightarrow 0} \omega^{2} \epsilon(\omega, \tau)
$$

if

$$
\lim _{\tau \rightarrow 0} \gamma(\tau) / \tau=0
$$

This happens for the example considered in Ref.[23] where at low temperatures, $\gamma(\tau) \propto \tau^{2}$. In this particular case, $\lim _{\tau \rightarrow 0} r(\alpha \tau, \tau)=r_{\text {plasma }}(0)$. As an immediate consequence, we can, 
in the general case, no longer perform the previous manipulations for all $n$. In fact, we have to treat $n=0$ separately.

The problem can be handled defining a new function $\tilde{g}$ which is identical to $g$ for all nonzero Matsubara frequencies, but does not feature the discontinuity discussed above. In the case of the Drude model this can be easily done with the substitution $g(0, \tau) \mapsto \tilde{g}(0)=$ $\lim _{\tau \rightarrow 0} g(\alpha \tau, \tau)$. The function $\tilde{g}$ so defined has the same analytical properties as a function $g$ with no temperature dependence, and we can therefore repeat the previous analysis. The substitution has to be made without quantitatively changing our expressions. This means that we have to rewrite the expression for the entropy given in Eq.(4) as follows

$S=-\left[\frac{g(0, \tau)-\tilde{g}(0)}{2}+\sum_{n=0}^{\infty}{ }^{\prime}\left[\tilde{g}(\mathrm{i} n \tau, \tau)+\mathrm{i} n \tau \tilde{g}_{\omega}(\mathrm{i} n \tau, \tau)+\tau \tilde{g}_{\tau}(\mathrm{i} n \tau, \tau)\right]\right]$

For the first two terms in the sum we can repeat the previous analysis and show that they vanish in the limit $\tau \rightarrow 0$. The last term in the sum can be approximated by

$$
\sum_{n=0}^{\infty}{ }^{\prime} \tau \tilde{g}_{\tau}(\mathrm{i} n \tau, \tau)=\partial_{\tau} \gamma(\tau) \sum_{n=0}^{\infty}{ }^{\prime} \tau \frac{\partial \tilde{g}(\mathrm{i} n \tau, \tau)}{\partial \gamma} \stackrel{\tau \rightarrow 0}{\longrightarrow} \partial_{\tau} \gamma(\tau) \int_{0}^{\infty} \frac{\partial \tilde{g}(\mathrm{i} \xi, \tau)}{\partial \gamma} d \xi
$$

which is equal to zero for an at least quadratic temperature dependence of $\gamma(\tau)$. In this case the low-temperature limit of the Casimir entropy is

$$
S=-\sum_{p, \mathbf{k}} \frac{g(0)-\tilde{g}(0)}{2}=\frac{1}{2} \sum_{\mathbf{k}} \tilde{g}_{\mathbf{k}}^{\mathrm{TE}}(0)<0
$$

where we have restored the sum over the wavevectors. Inspection shows that this is nothing but the result of Bezerra et al.[23].

\section{A sum over modes in the dissipative case}

One of the difficulties in generalizing Eq.(1) to a dissipative system is the concept of a "mode" because this represents a state of constant energy which can be well defined only for a closed system where dissipation does not occur. The naively constructed complex mode frequencies actually do not represent the real modes of our system. In fact, in quantum mechanics a dissipative system is by definition an open one [24, 25, 26, 27, 28, 29], i.e. a system coupled a macroscopic system, generally a thermal bath. The bath contains a continuum of degrees of freedom into which the system energy can be irreversibly dissipated. A formula like Eq.(1) is applicable only if we consider the modes of the total system - which is, of course, cumbersome or plainly impossible in practice [30]. It is natural to ask, however, whether it may be useful to apply the familiar picture of a damped oscillator in terms of a complex frequency, which is just the pole of the Green function for this system. Here we are going to show that this compact description can be applied to the Casimir energy as well, with the complex poles going over into conventional modes in the non-dissipative case.

\subsection{Sum over poles}

Our starting point will be the Lifshitz formula for the Casimir force between two slabs with finite thickness [7, 31, 32, 33]

$$
F=\frac{\hbar}{4 \pi} \sum_{p, \mathbf{k}} \partial_{L} \int_{-\infty}^{\infty} d \omega \operatorname{coth}\left[\frac{\pi \omega}{\tau}\right] \operatorname{Im}\left[\ln D_{\mathbf{k}}^{p}(\omega)\right]_{\infty}^{L}
$$


where the frequency integral is just above the real axis $(\operatorname{Im} \omega>0)$ ensuring retarded response functions. The environment is at the scaled temperature $\tau$ (see Eq. (3)). We recall the renormalization, $[\cdots]_{\infty}^{L}$, with respect to a pair of infinitely distant mirrors. In the following, we drop again the sum over $p$ and $\mathbf{k}$. Finally, the relevant dispersion function is

$$
\partial_{L} \ln D(\omega)=\kappa \frac{1+r(\omega)^{2} \mathrm{e}^{-2 \kappa L}}{1-r(\omega)^{2} \mathrm{e}^{-2 \kappa L}}
$$

with $r(\omega)$ being the reflection coefficient of the mirrors (supposed identical for simplicity). We used the reality of the optical response, $r(\omega)^{*}=r\left(-\omega^{*}\right)$, to extend the integral (20) to the negative frequency axis. After a few manipulations, the Casimir energy can be written

$$
E=\frac{\hbar}{2} \sum_{p, \mathbf{k}} \Omega_{\mathbf{k}}^{p}, \quad \Omega=-\int_{-\infty}^{\infty} \frac{d \omega}{2 \pi} \omega \operatorname{coth}\left[\frac{\pi \omega}{\tau}\right] \operatorname{Im}\left[\partial_{\omega} \ln D(\omega)\right]_{\infty}^{L}
$$

The integrand of this equation goes to zero sufficiently fast for $|\omega| \rightarrow \infty$ so that we can close the integral in the complex plane along a path which encloses the lower half-plane in the clockwise sense..

The function $D$ is constructed in such a way that its zeros are the complex "mode" frequencies we are looking for. But, $D$ also shows branch cuts along the real axis that prevent a straightforward deformation of the integration contour. These branch cuts come in two types: the first corresponds to the mode continuum incident from outside the cavity; the second is connected with the dissipative phenomenon and arises from the continuum of modes describing the bath. Following Ref.[16], we eliminate the branch cut of the first type by placing our system into a perfectly reflection box with a length $a>L$. The second type of cut is avoided by analytically continuing $D$ from the upper half to the lower half of the complex frequency plane. It is exactly because of this step that the zeros of $D$, otherwise located on a unphysical sheet, become apparent [30]. With these changes, $D$ turns into a function $D_{a}$ that shows, instead of a branch cut, a set of discrete zeros that adds to the ones of $D$. One can proceed in the usual way with $D_{a}$ and take the limit $a \rightarrow \infty$ at the end of the calculation.

All functions involved in the integral (22) are now meromorphic functions [34] and can be expanded over their singularities as follows

$$
\operatorname{coth}\left[\frac{\pi \omega}{\tau}\right]=\sum_{n=-\infty}^{\infty} \frac{\tau / \pi}{\omega+\mathrm{i} n \tau}, \quad \partial_{\omega} \ln D_{a}(\omega)=\sum_{m}\left(\frac{1}{\omega-\omega_{m}}+\frac{1}{\omega+\omega_{m}^{*}}\right)
$$

The zeros of $D_{a}$ must satisfy $\operatorname{Im} \omega_{m}<0$ because of causality and passivity. Moreover, the dispersion function inherits the reality condition, $D_{a}(\omega)^{*}=D_{a}\left(-\omega^{*}\right)$, of the reflection coefficients. Therefore, the zeros occur in pairs, $\omega_{m}$ and $-\omega_{m}^{*}$, on both sides of the imaginary axis.

With a little algebra and exploiting the residues theorem one can show

$\Omega=\operatorname{Re}\left[\sum_{m} \omega_{m} \operatorname{coth}\left[\frac{\pi \omega_{m}}{\tau}\right]\right]_{L \rightarrow \infty}^{L}+\operatorname{Re}\left[\sum_{m} \sum_{n=0}^{\infty} \frac{\mathrm{i} \omega_{m}}{\pi} \frac{2 n \omega_{T}^{2}}{(n \tau)^{2}+\omega_{m}^{2}}\right]_{\infty}^{L}$

where we have already taken the limit $a \rightarrow \infty$. Note also the following identity

$$
\operatorname{Im}\left[\sum_{m} \omega_{m}\right]_{\infty}^{L}=\int_{-\infty}^{\infty} d \omega \omega \operatorname{Im}\left[\partial_{\omega} \ln D\right]_{\infty}^{L}=0
$$

than can be easily shown because of parity. This sum rule ensures that in the sum over $n$ in Eq.(24), the summands go like $1 / n^{2}$ for $n \rightarrow \infty$. 
As a check let us consider the non-dissipative case: the frequencies $\omega_{m}$ are then real, the second term in Eq.(24) vanishes, and the Casimir energy can be written as

$$
E=\frac{\hbar}{2} \sum_{p, \mathbf{k}}\left[\sum_{m} \omega_{m}\left(2 \bar{n}_{T}\left(\omega_{m}\right)+1\right)\right]_{\infty}^{L}
$$

where $\bar{n}_{T}\left(\omega_{m}\right)$ is just the mean number of thermal photons in the frequency mode $\omega_{m}$. In the limit of zero temperature this number goes to zero and we get the expressions of Ref. [35, 36].

In the dissipative case, we focus on the zero temperature limit $(\tau \rightarrow 0)$ : the hyperbolic cotangent in Eq.(24) goes to unity, while the second term becomes an integral so that we have

$$
\tau \rightarrow 0: \quad \Omega \rightarrow \operatorname{Re}\left[\sum_{m} \omega_{m}\right]_{\infty}^{L}+\int_{0}^{\infty} d \xi \operatorname{Re}\left[\sum_{m} \frac{\mathrm{i} \omega_{m}}{\pi} \frac{2 \xi}{\xi^{2}+\omega_{m}^{2}}\right]_{\infty}^{L}
$$

The integral converges at the upper limit thanks to the sum rule (25) and we can write

$$
E=\frac{\hbar}{2} \sum_{p, \mathbf{k}} \operatorname{Re}\left[\sum_{m}\left(\omega_{m}-\frac{2 \mathrm{i} \omega_{m}}{\pi} \ln \frac{\omega_{m}}{\Lambda}\right)\right]_{\infty}^{L}
$$

where the meaning of the constant frequency $\Lambda$ will become clear soon. This is the main result of this section: we have found the Casimir energy between dissipative mirrors, at zero temperature, as a sum over cavity mode frequencies. The expression is valid for a generic (causal) dielectric function and for mirrors with arbitrary thickness. One sees immediately that the non-dissipative case reduces to the standard sum over real mode frequencies.

To interpret the two terms in Eq.(28), observe that for each collection of quantum numbers $p, \mathbf{k}, m$, we deal with the average energy of a quantum damped harmonic oscillator, as discussed in detail by Nagaev and Büttiker [37]. The first term of the energy is the familiar zero point energy, renormalized by the coupling to the environment (see also Ref.[29]). The second part of the energy is the most interesting one. It arises because the ground state of the oscillator is no longer an eigenstate of the whole oscillator+bath system. (This can be traced back to antiresonant terms in the coupling Hamiltonian.) As a consequence, the oscillator energy is subject to quantum fluctuations, whose average leads to an extra energy with a logarithmic behavior. Within this analogy, the constant $\Lambda$ takes the role of a cutoff frequency for the coupling to the bath. In fact, for a single oscillator, the cutoff is needed to renormalize some quantities like the momentum fluctuations [37]. It is interesting to note that for the Casimir energy, the sum rule (25) removes the cutoff dependence in the final result.

\section{Conclusions}

We have explored the impact of dissipation and of temperature on the Casimir effect. On fairly general grounds, we have demonstrated that the Casimir entropy vanishes at $T \rightarrow 0$ (the Nernst theorem is satisfied) when the material properties (reflection coefficients) behave continuously as both frequency and temperature are decreased to zero. A discontinuity in the plane of these variables leads to a non-vanishing, typically negative entropy. We have argued that this can be traced to the behavior of the TE reflection coefficient at low frequencies. Both results are in agreement with previous work addressing specific models. The complex mode frequencies that occur between dissipative slabs are finally a useful tool to compute the Casimir energy as a generalized sum over "modes", although the mode concept has to be treated with care in this intrinsically open system. Our expression Eq.(28) shows that the Lifshitz formula (that we started with) does not reduce to the "recipe" of taking the real part 
of a complex sum over modes, as stated in Refs. [17, [18]. The latter sum deeply changes its structure if dissipation is allowed for, while the Lifshitz expression remains formally the same. We have argued that the logarithmic correction that we find has a clear meaning from a physical point of view, by drawing the analogy to a damped quantum oscillator. As it happens, the result is independent of the cutoff frequency for the coupling to the environment.

\section{Acknowledgments}

F. Intravaia thanks the Alexander von Humboldt Foundation for financial support.

\section{References}

[1] H. Casimir, Proc. kon. Ned. Ak. Wet 51, 793 (1948).

[2] U. Mohideen and A. Roy, Phys. Rev. Lett. 81, 4549 (1998).

[3] G. Bressi, G. Carugno, R. Onofrio, and G. Ruoso, Phys. Rev. Lett. 88, 041804 (2002).

[4] H. B. Chan, V. A. Aksyuk, R. N. Kleiman, D. J. Bishop, and F. Capasso, Phys. Rev. Lett. 87, 211801 (2001).

[5] A. Lambrecht and S. Reynaud, in Poincare Seminar 2002: Vacuum Energy-Renormalization, Vol. 30 of Progress in Mathematical Physics, edited by V.Rivasseau and B.Duplantier (Birkhauser, Basel, 2003), p. 109.

[6] R. Onofrio, New J. Phys. 8, 237 (2006).

[7] E. Lifshitz, Sov. Phys.-JETP (USA) 2, 73 (1956).

[8] M. Boström and B. E. Sernelius, Phys. Rev. Lett. 84, 4757 (2000).

[9] G. Klimchitskaya and V. Mostepanenko, Phys. Rev. A 63, 062108 (2001).

[10] V. Bezerra, G. Klimchitskaya, and V. Mostepanenko, Phys. Rev. A 65, 052113 (2002).

[11] V. B. Svetovoy and M. V. Lokhanin, Phys. Rev. A 67, 022113 (2003).

[12] I. Brevik, J. B. Aarseth, J. S. Hoye, and K. A. Milton, in Proc. 6th Workshop on Quantum Field Theory under the Influence of External Conditions, edited by K. A. Milton (Rinton Press, Paramus, NJ, 2003).

[13] V. M. Mostepanenko et al., J. Phys. A 39, 6589 (2006).

[14] B. E. Sernelius, J. Phys. A 39, 6741 (2006).

[15] V. B. Svetovoy and R. Esquivel, J. Phys. A 39, 6777 (2006).

[16] K. Schram, Phys. Lett. A 43, 282 (1973).

[17] D. Langbein, Phys. Rev. B 2, 3371 (1970).

[18] D. Langbein, Theory of Van der Waals attraction, Vol. 72 of Springer Tracts in Modern Physics (Springer, Berlin, 1974).

[19] J. Schwinger, L. L. DeRaad, Jr., and K. A. Milton, Ann. Phys. 115, 1 (1978).

[20] M. Abramowitz and I. Stengun, Handbook of Mathematical Functions (Dover Books, New York, 1971).

[21] M. Boström and B. E. Sernelius, Physica A 339, 53 (2004).

[22] V. B. Svetovoy and R. Esquivel, Phys. Rev. E 72, 036113 (2005).

[23] V. B. Bezerra, G. L. Klimchitskaya, V. M. Mostepanenko, and C. Romero, Phys. Rev. A 69, 022119 (2004).

[24] A. O. Caldeira and A. J. Leggett, Phys. Rev. Lett. 46, 211 (1981).

[25] A. O. Caldeira and A. J. Leggett, Ann. Phys. (N.Y.) 149, 374 (1983).

[26] H. Breuer and F. Petruccione, The Theory of Open Quantum Systems (Oxford University Press, Oxford, 2002).

[27] G. W. Ford, M. Kac, and P. Mazur, J. Math. Phys. 6, 504 (1965).

[28] G. W. Ford and M. Kac, J. Stat. Phys. 46, 803 (1987).

[29] F. Intravaia, S. Maniscalco, and A. Messina, Phys. Rev. A 67, 042108 (2003).

[30] B. E. Sernelius, Phys. Rev. B 74, 233103 (2006).

[31] M.-T. Jaekel and S. Reynaud, J. de Phys. I (France) 1, 1395 (1991).

[32] C. Genet, Ph.D. thesis, Univ. Paris VI, 2002.

[33] C. Genet, A. Lambrecht, and S. Reynaud, Phys. Rev. A 67, 043811 (2003).

[34] A. I. Markusevic, Elements of Theory of Analytic Functions (Mir, Moscow, 1988).

[35] F. Intravaia and A. Lambrecht, Phys. Rev. Lett. 94, 110404 (2005).

[36] F. Intravaia, C. Henkel, and A. Lambrecht, Phys. Rev. A 76, 033820 (2007).

[37] K. E. Nagaev and M. Büttiker, Europhys. Lett. 58, 475 (2002). 